\title{
URBAN SPATIAL ECOLOGICAL PERFORMANCE BASED ON THE DATA OF REMOTE SENSING OF GUYUAN
}

\author{
REN Xiao-juan ${ }^{1, *}$, CHEN Xiao-jian ${ }^{1}$, MA Quan ${ }^{2}$ \\ ${ }^{1}$ College of Architecture, Xi'an University of Architecture and Technology, Xi'an 710055, China- msren982243325@163.com.cn \\ ${ }^{2}$ The First Institute of Photogrammetry and Remote Sensing, NASG, Xi'an 710054, China-121814807@qq.com
}

Commission III, WG III/7

KEY WORDS: Urban Spatial Ecological Performance; Urban Landuse; High-resolution Remote Sensing Data;

\begin{abstract}
:
The evolution analysis of urban landuse and spatial ecological performance are necessary and useful to recognizing the stage of urban development and revealing the regularity and connotation of urban spatial expansion. Moreover, it lies in the core that should be exmined in the urban sustainable development. In this paper, detailed information has been acquired from the high-resolution satellite imageries of Guyuan, China case study. With the support of GIS, the land-use mapping information and the land cover changes are analyzed, and the process of urban spatial ecological performance evolution by the hierarchical methodology is explored. Results demonstrate that in the past 11 years, the urban spatial ecological performance show an improved process with the dramatic landcover change in Guyuan. Firstly, the landuse structure of Guyuan changes significantly and shows an obvious stage characteristic. Secondly, the urban ecological performance of Guyuan continues to be optimized over the 11 years. Thirdly, the findings suggest that a dynamic monitoring mechanism of urban land use based on high-resolution remote sensing data should be established in urban development, and the rational development of urban land use should be guided by the spatial ecological performance as the basic value orientation.
\end{abstract}

\section{METHODOLOGY AND DATA SOURCE OF RESEARCH}

\subsection{Overview of the Study Area}

Guyuan is a regional central city in the south of Ningxia with prominent contradiction between people and land. Since the establishment of the city in 2002, the economic structure of Guyuan has been continuously transformed, the economic growth rate has been constantly rising, and the urban space has changed from a single center to a multi-group with the expansion trend of "leapfrog". According to the map information of the building area extracted from the remote sensing images of Guyuan city, combined with the quantitative calculation of the related indexes of urban spatial expansion, it is found that the spatial expansion of urban construction land in Guyuan's shows a typical periodicity and a clear direction of expansion. For the complication of morphological structure and the incoordination of elasticity between population growth and land expansion(REN Xiaojuan, 2017 ), it is typical to study the ecological performance of spatial expansion in Guyuan.

\subsection{Data Sources and Technical Routes}

Taking Guyuan City as the case, the study takes the moderately expanded area of urban land use in Guyuan City in 2014 as the object, with a total area of approximately $52.68 \mathrm{~km}^{2}$. Based on the GIS operation platform and three typical periods since the establishment of the city of Guyuan, the study obtains vectorized maps of the urban areas from the high-resolution remote sensing images in 2003, 2009, and 2014(Table 1). Combined with the investigation data and related statistical data, the urban spatial ecological performance in the period of rapid expansion of Guyuan City is calculated visually and quantitatively.

\begin{tabular}{|c|c|c|}
\hline Satellite Name & Spatial Resolution (m) & Archiving Date \\
\hline QuickBird (QB) & 0.61 & Sep. 4, 2003 \\
QuickBird (QB) & 0.61 & Jun 9, 2009 \\
GF-1,China & 2.0 & May 19,2014 \\
\hline
\end{tabular}

Table 2. Basic characteristics of remote sensing data

\subsection{Methodology of Research}

The research on the combination of qualitative and quantitative research under the support of spatial analysis technology is used to research the ecological performance of Guyuan's spatial expansion. The research first combines the existing research results(Li Lan, 2017), and adopts a hierarchical deconstruction method to solve the complexity of urban spatial expansion, and quantitatively analyzes the ecological performance of spatial expansion of Guyuan City through four levels of indicators (Figure1 and Figure 2). The four levels are the ecoenvironmental adaptive performance layer, the ecological structure stability performance layer, the eco-landscape continuity performance layer, and the eco-service function performance layer. After the quantitative study, the indicators were subjectively and objectively combined and grid computing was performed to obtain the performance evaluation results of the corresponding evaluation layer. The method is good at reflecting the disturbance of spatial ecological performance and the spatial, spatial and temporal coupling characteristics of spatial ecological performance in the expansion process of

\footnotetext{
* Corresponding author
} 
urban space, and is convenient for quantitative and spatial visualization research.

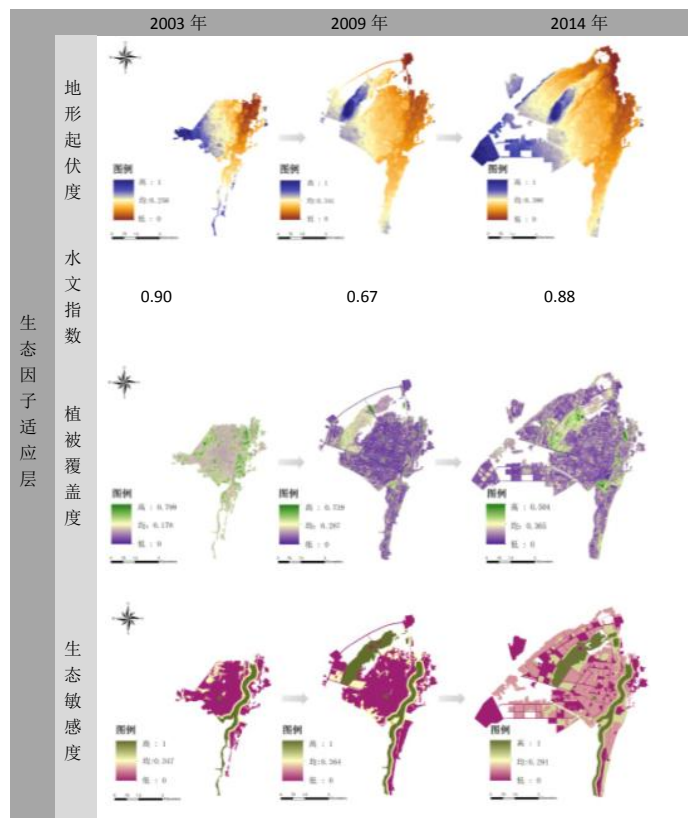

Figure 1. The extraction process of the ecological environment adaptive performance factor in Guyuan City

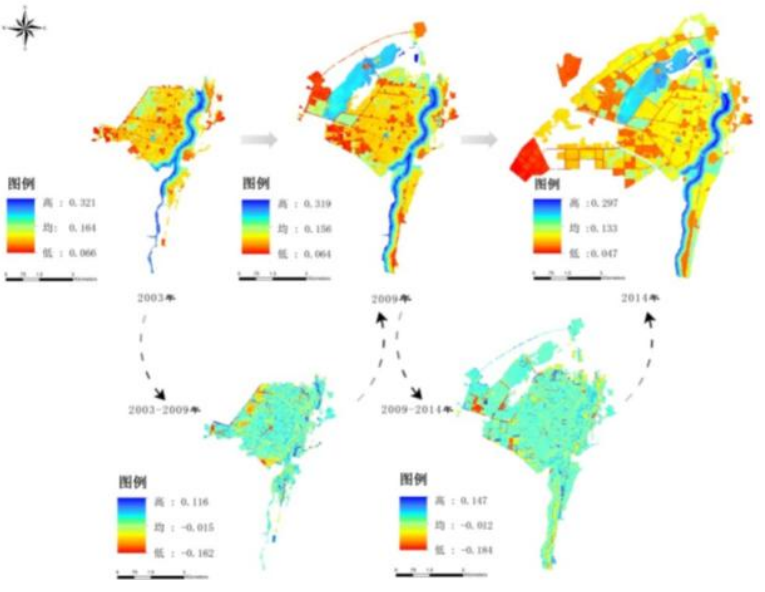

Figure 2. Analysis of evolvement of ecological environment adaptability performance in Guyuan City from 2003 to 2014

\section{THE LAND USE ECOLUTION IN GUYUAN CITY}

\subsection{Evolution of Land use}

From 2003 to 2014, the difference in land use structure in Guyuan City continues to increase. In particular, the area of agro-forestry land decreases as well as the area of urban construction land increases sharply, the latter replaces the former as the substrate (Figure 3). From 2003 to 2014, the net output from agricultural and forestry land is $2180.75 \mathrm{hm} 2$, and the average annual output is $198.25 \mathrm{hm} 2$. The variability of the dominant status of agricultural and forest lands and urban land reflects the typical shortcomings of heavy space expansion and protection of light agriculture and forest land at the stage of rapid urban development.

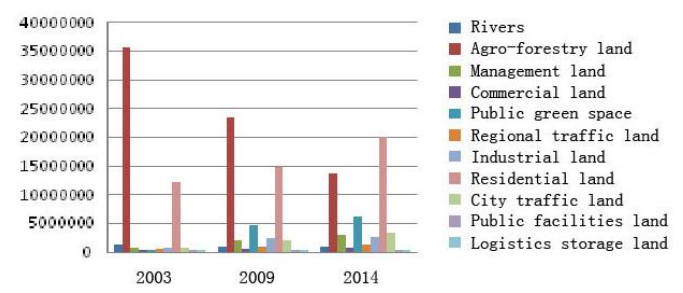

Figure 3. Land Use Structure of Guyuan City, 2003-2014

\subsection{Typical problems of land use evolution}

Based on the map information of the construction areas of Guyuan City extracted from remote sensing images and the nonagricultural population of the municipal districts from the "CHINA CITY STATISTICAL YEARBOOK" in relevant years

(National Bureau of statistics, 2001-2015), the urban spatial expansion elasticity index of Guyuan City is calculated. At the beginning of the study, the coefficient of urban extensional elasticity was 2.054 , indicating that there was a surplus in the urban scale during this period, and then the expansion was further accelerated. By 2007, it reached an ultra-high value of 8.277 , and the scale of the city had exceeded the reasonable value; the coefficient of elasticity was reduced to 0.023 by 2009. ; It rose to 5.433 in 2014. During the expansion of urban space in Guyuan City, the elasticity coefficient of the extreme changes should be vigilant. Except for the minimum value of the expansion elasticity index in 2009, the elasticity index of the built-up areas in Guyuan City in other research years has exceeded the reasonable urban spatial expansion elasticity index. This has exposed the problem of imbalances in the expansion of urban space.

\section{THE EVOLUTION OF ECOLOGICAL PERFORMANCE IN GUYUAN CITY}

According to the weighted and superimposed operation of raster data, the results of performance evaluation of the corresponding evaluation levels and the main points of view are obtained. In order to be easy to describe, Guyuan is divided into four main urban areas according to the characteristics of the urban morphology and the expansion order. The district A is the main component of the established area in 2003. Area B is the extension area of 2003-2009, area C and D is for 2009-2014. In addition, there are still a few expansion areas in the east of the Qingshui River (Figure 4).

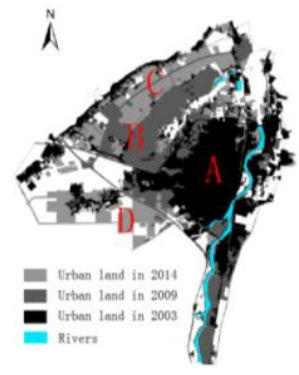

Figure 4. Diagram of evaluation partition 


\subsection{The indicators Evolution of Ecological Performance at all levels}

3.1.1 Analysis and evaluation of ecological environment adaptability: From 2003 to 2014, the performance level of ecological environment adaptability in Guyuan city has decreased significantly from 0.164 in 2003 to 0.133 in 2014 , which has decreased by $4.9 \%$ and $14.0 \%$ respectively.

In the previous phase, the adaptive performance level of the original urban area has decreased by $9.1 \%$, and the spatial distribution is symmetrical. But a low-value zonal area forms in the west, and the performance of the extended area exceeds the original urban area level of $9.9 \%$. The insufficient accumulation of performance in the original urban area is the main reason for the lower performance value in 2009 than in 2003. In the latter period, the performance level of the original urban area has decreased by $7.5 \%$, and the overall performance of the expansion area is lower than the average level of urban performance in 2014. The performance level of the district $\mathrm{C}$ and $\mathrm{D}$ are $9.3 \%$ and $30.2 \%$ lower than that of the 2003 level, respectively for insufficient accumulation of original performance and rapid expansion of the new area. From the spatial differentiation, the performance of area B is always higher than the overall level of the corresponding study period. Qingshui River and Guyan Mountain are the main distribution areas to maintain high ecological adaptability.

3.1.2 Analysis and evaluation of Ecological structure stability: After a certain fluctuation, the performance level of ecological structure stability in Guyuan city has decreased by $4.2 \%$ and $3.0 \%$ respectively from 2003 to 2014 .

The performance level of structural stability in the original urban area has decreased by $13.9 \%$ on average, and the performance level of extended area is better than that of original urban area and the average level of city as a whole. The lack of accumulation in the original urban area is the direct reason for lowering the overall level of the city. In the base year of the study, two high-performance distribution zone forms respectively on the east and west of the urban, which converge in the southern part of the city and decrease in 2009. A new high-energy group forms in the extended area (Guyan Mountain). In the latter period, the performance level in 2014 is better than the performance level in 2009. Among them, the performance level of the original urban area has improved by $1.1 \%$ than the average level of the city. In the spatial distribution, the southwest group and Guyan Mountain region are the main areas of high efficiency.

3.1.3 Analysis and evaluation of Ecological process continuity: The performance of ecological process continuity fluctuates, indicating that the continuity of urban landscape patterns and structures is disturbed by Urban spatial expansion. In the previous period, the performance level in 2009 is 5.6 lower than that in 2003, and the latter is higher in 2014 than that in 2009, but it is still 3.2 lower than that in 2003(Table 2).

\begin{tabular}{|l|l|l|l|}
\hline Performance value of the & 2003 & 2009 & 2014 \\
\cline { 2 - 4 } ecological process continuity & 0.125 & 0.118 & 0.121 \\
\hline
\end{tabular}

Table 2. Performance value of the ecological process continuity in Guyuan during 2003-2014
3.1.4 Analysis and evaluation of Ecosystem service performance: The service performance of ecosystem has increased by 2.24 times and $76.2 \%$ respectively from 0.021 in 2003 to 0.084 in 2014 .

During the previous expansion period, the service performance of the original urban area in 2009 is 2.25 times higher than that in 2003. The level of the expansion area reaches the average level of the city. In the newer extension area, the performance level of the original urban area has increased by $76.2 \%$, the expansion level of district $\mathrm{C}$ is lower $0.4 \%$ than that of the average level in 2003, and district D is higher $5.9 \%$ than that in 2003. The improvement of the performance level of the original urban area is the main reason for the improvement of the urban ecosystem service performance, and the contribution value of Guyan Mountain and Qingshui River are the highest in terms of spatial distribution.

\subsection{The Evolution of Ecological Performance at the whole level}

3.2.1 Analysis the Ecological Performance at the whole level: The performance level of the ecosystem in Guyuan City is in an increasing process, with a large increase of $72.1 \%$ in the period from 2003 to 2009 , and the performance level reaches 0.378 in 2014 which is higher $29.3 \%$ than that in 2003. The Qingshui River Basin and the Guyan Mountain with large area of continuous ecological land are distribution areas with higher value of urban ecosystem performance.

According to the spatial evolution process, the urban performance of the original urban area has increased by $71.8 \%$ in the previous period, and the performance level of the extended area reached the average level of the city. In the latter period, the performance of the original urban area has increased by $29.8 \%$ than that in 2003, and the performance of the extended area is lower than the average level of the city in 2014. The improvement of the performance level in the original urban area contributes significantly to the whole performance level, showing characteristics of internal superior to external (Figure 5).

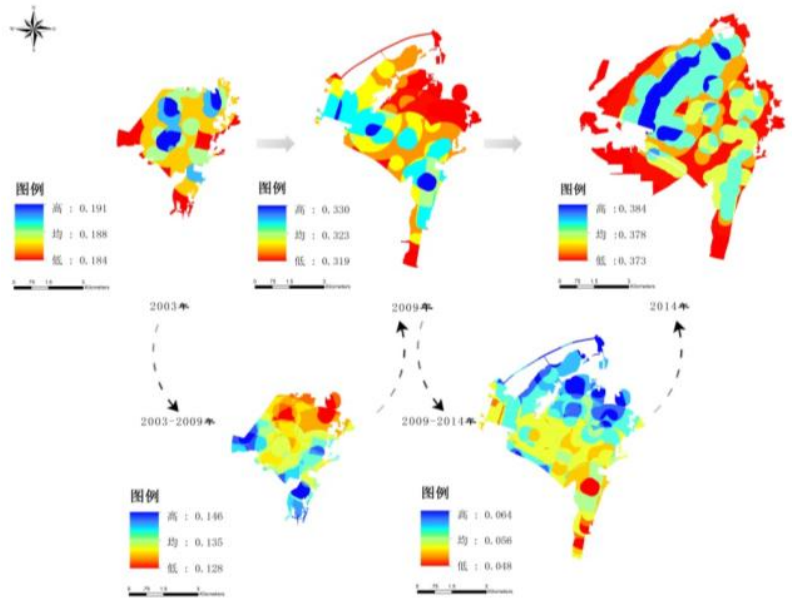

Figure 5. Analysis of Spatial dynamic changes of Ecological performance in Guyuan City from 2003 to 2014 
3.2.2 Evaluation the Ecological Performance at the whole level: Judging from the development process, the spatial expansion in the previous period is larger than that in the later, because the scale of the land with ecological value in the previous period increases significantly in the scope of the study. And in the latter period, with the acceleration of the spatial expansion, the increase of artificial land is obvious, the growth rate of green space is smaller than the previous period.

From the perspective of the change characteristics of the internal indicators in the system, the change of the internal indicators of the system is basically the same tendency of reduction, except for the significant improvement of the service performance level of the ecosystem. The service performance level of urban ecosystem contributes significantly to the improvement of urban ecosystem performance, which indicates that urban spatial expansion disturbs urban ecosystem. However, with the increase of the area of urban ecological green space, it can effectively provide external ecological service value and maintain ecological benefit.

\section{IDENTIFICATION OF THE ECOLOGICAL STRUCTURE OF GUYUAN CITY BASED ON THE EVOLUTION OF SPATIAL ECOLOGICAL PERFORMANCE}

Under the influence of natural and urban spatial pattern, with the Guyanling region being brought into the development frame of central urban area, the ecological value performance of Guyuan city is improved for the enhancement of external service value of ecosystem. At the same time, the main spatial structure is shown as "one mind and two belts". Therefore, full protection and utilization of the ecological sensitive resources such as mountains and rivers are useful to improve the ecological performance. Meanwhile, increasing the area of green space and distributing it orderly in space are also benefit to the eco-performance. At the same time, it is advantageous to introduce the airflow into the central area of the city through the development of ecological green axis, since ensuring the environmental requirements of urban ventilation is a beneficial urban ecological pattern (Figure 6).

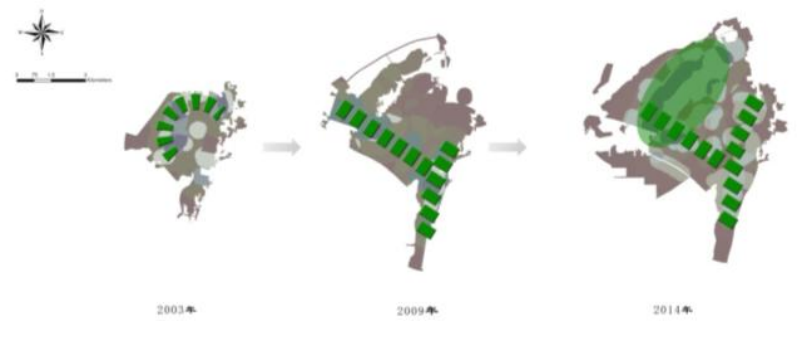

Figure 6. Spatial structure Evolution of Ecological performance in Guyuan City

\section{CONCLUSION}

With the support of GIS technology, the high-resolution remote sensing satellite images as the basic data source are used to study the evolution of eco-performance in Guyuan in real time and objectively. The high-resolution remote sensing data show significant advantages for dynamic, quantitative, and visual examining of urban landuse change and urban spatial ecological performace evolution.
After the foundation of Guyuan, it is reciprocal causation that the structure of land-use changes obvious and the urban construction land engulfs to natural land rapidly. The dominant position of agroforestry land in the early is replaced by urban construction land in the later stage of development. It reflects the typical disadvantages of emphasizing space expansion and neglecting the protection of agroforestry land. Meanwhile the substitution process of construction land for agroforestry land reflects serious problems of excessive expansion and imbalance of expansion elasticity.

The preservation of large areas of ecological land and the spatial integration with the urban built environment are the main reasons for the improvement of the spatial ecological performance of Guyuan City. To protect and utilize ecologically sensitive resources such as mountains and water, and incorporate them into the ecological spatial structure as well as to increase the area of green space and distribute them in an orderly manner in space are conducive to improving the ecoperformance.

Finally, it is suggested that a dynamic detection mechanism based on spatial ecological performance and remote sensing data should be established in urban development to guide the rational development of cities.

\section{ACKNOWLEDGEMENTS}

The work is funded by National Natural Science Foundation of China(NO.51378420). The authors would thank the editor and the anonymous reviewers for their precious time in reviewing this manuscript and their valuable comments, suggestions, and editing.

\section{REFERENCES}

Li Lan, 2017. Interpretation of Urban Space: Subject Value and Expansion Performance. China Architecture \& Building Press, Beijing, China.

National Bureau of statistics, 2001-2015. China urban statistical yearbook. China Statistical Press, Beijing, China.

Ren Xiao-juan, Chen Xiao-jian, Ma Quan, 2017. Urban Spatial expansion and motivation Analysis in Northwest China: Taking Guyuan City of Ningxia as a Case. Remote Sensing Information, (3), pp.155-161.

Revised April 2018 